the DNA samples and recording relevant clinical features. When papers describing mutations in genes responsible for human diseases are published, clinical details must be there. Experience tells us that if the full clinical descriptions have not been collected and recorded on the affected individuals whose DNA has led to identification of a mutation, they never will be (it is very difficult to contact people again). Authors need to insist that the clinical information collected by their colleagues be published or at the very least be readily accessible. Ideally, it should be part of every publication, although an accessible website is another option. If this information is not available, opportunities to advance our understanding of biology and human disease and to help affected individuals will be lost.

1. Amiel, J. et al. Nat. Genet. 33, 459-461 (2003) 2. Dodé, C. et al. Nat. Genet. 33, 463-465 (2003).
3. Fantes, J. et al. Nat. Genet. 33, 461-463 (2003)

4. Haug, K. et al. Nat. Genet. 33, 527-532 (2003)

5. O'Driscoll, M., Ruiz-Perez, V.L., Woods, C.G., Jeggo, P.A. \& Goodship, J.A. Nat. Genet. 33, 467-501 (2003).

6. Puls, I. et al. Nat. Genet. 33, 455-456 (2003).

7. Robertson, S.P. et al. Nat. Genet. 33, 487-491 (2003).

8. Slager, R.E., Newton, T.L., Vlangos, C.N., Finucane, B. \& Elsea, S.H. Nat. Genet. 33, 466-468 (2003).

9. Majewski, F., Ranke, M. \& Schinzel, A. Am. J. Med. Genet. 12, 23-35 (1982).

10. Hall, J.G., Froster-Iskenius, U.G. \& Allanson, J.E, Handbook of Normal Physical Measurements (Oxford Medical Publications, Oxford, 1989).

\title{
The smoking gun of gene transfer
}

Gene transfers from chloroplasts to the nucleus occur naturally over evolutionary time scales but have always been inferred from sequence comparisons, never directly observed. A notable new report has caught plastid-to-nucleus gene transfer in the act, providing exciting opportunities to study its mechanisitic details in the laboratory and its ecological implications in nature.

About 1.5 billion years ago, a free-living cyanobacterium took up permanent residence in a eukaryotic host cell. The results of that symbiosis are today's chloroplasts, the photosynthesizing organelles of plants. One of the most important processes that occurred en route to the establishment of the chloroplast as a stably inherited organelle (as opposed to a transient endosymbiont) was the transfer of genes from the cyanobacterial symbiont's genome to the host's nuclear chromosomes. Recent estimates suggest that as much as $18 \%$ of the nuclear genes in Arabidopsis thaliana ultimately stem from the ancestral plastid genome. But gene transfer from chloroplasts to the nucleus has always been indirectly inferred from genesequence comparisons-until now. In a recent report, chloroplast-to-nucleus gene transfer was observed in flagrante, capturing what may be nature's most prevalent kind of genetic promiscuity: lateral gene transfer from organelles to the nucleus.

Using experimental techniques pioneered by Pal Maliga, Huang et al. (Nature 422, 72-76 (2003)) introduced a fragment of foreign DNA into the chloroplast genome of tobacco, Nicotiana tabacum. The fragment, which was stably integrated into the circular chloroplast chromosome by homologous recombination, contained an antibiotic resistance marker that is expressed in the chloroplast. This allowed them to obtain

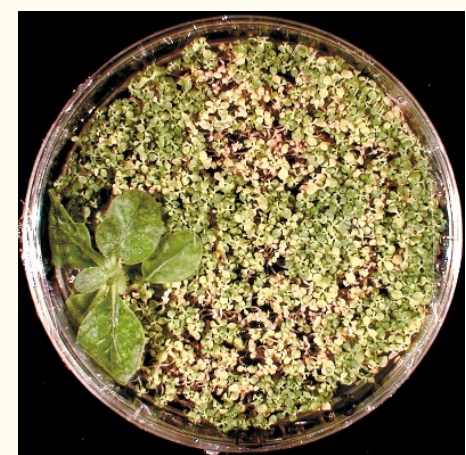

A rare (1 in 16,000) kanamycin-resistant plant (photo courtesy of J. Timmis).

plants whose plastids - but only their plastids-were homogeneously transgenic (homoplastomic transformants). Their decisive trick was then simple: in addition to the plastid-specific resistance marker, their chloroplast DNA-integrated fragment also contained a kanamycin-resistance gene, which possessed a spliceosomal intron under the control of promoter specific to nuclear gene expression. These homoplastomic lines were used as the male (pollen) donor in crosses to wild-type female recipients. The resulting seed was sown on medium containing kanamycin. The expectation was that only such seedlings would survive in which the kanamycin-resistance marker specific to the nuclear expression machinery had been transferred in a functional state from the chloroplast to the nuclear genome. Among 250,000 progeny tested, they found 16 independen kanamycin-resistant plants (see figure) with heritable nuclear insertions of the chloroplast fragment, which in most cases segregated in a mendelian fashion. That translates to a rate of successful chloroplastto-nucleus gene transfer accompanied by stable nuclear expression of one in 16,000 pollen grains tested.
As the authors point out, this surprisingly high frequency of gametes that acquired a fragment of chloroplast DNA is still an underestimate for the overall transfer rate of bulk DNA from the chloroplast to the nucleus, because only transfers of the engineered resistance gene that resulted in proper expression were detected. Transfers that did not encompass the region of chloroplast DNA into which the resistance gene had been inserted also went undetected. Hence, the true rate of bulk chloroplast DNA transfer is probably higher. On the other hand, N. tabacum is an allotetraploid, a circumstance that may buffer the potentially deleterious effects of large DNA insertions in the nuclear chromosomes, thus potentially permitting a higher transfer rate than might be expected in diploids. Yet there is no evidence one way or the other to suggest that the chloroplast-derived fragments are being inserted into active genes. Further study of the integration sites, their surrounding regions and the tendency of the integrated DNA to undergo deletion should provide additional clues.

The findings suggest that one in every few thousand plants that we see in the greenhouse, in cultivated fields or in nature has a large and freshly incorporated piece of chloroplast DNA somewhere in its nuclear chromosomes that was integrated only one generation ago. Given the geological time scales over which this rapid and efficient intracellular gene transfer mechanism has been operating, the biggest surprise of all is perhaps that there is any chloroplast-specific DNA left at all-but that is another story. -William Martin

Institute of Botany III, University of Dusseldorf, Dusseldorf, Germany.e-mail: w.martin@ uni-duesseldorf.de 